\title{
A Pulse Signal Characteristic Recognition Algorithm Based on Multifractal Dimension
}

\author{
Yi-bing Li, Wei Nie, Fang Ye, and Jing-chao Li \\ College of Information and Communication Engineering, Harbin Engineering University, Harbin 150001, China \\ Correspondence should be addressed to Fang Ye; yefang0815@sina.cn
}

Received 18 August 2014; Revised 15 November 2014; Accepted 15 November 2014; Published 9 December 2014

Academic Editor: Miguel A. F. Sanjuan

Copyright (C) 2014 Yi-bing Li et al. This is an open access article distributed under the Creative Commons Attribution License, which permits unrestricted use, distribution, and reproduction in any medium, provided the original work is properly cited.

In low SNR condition, it is difficult to identify the radio transient characteristics of the signals. To solve this problem, a new recognition algorithm based on multifractal dimension characteristics is proposed. In fractal theory, multifractal dimension is the most sophisticated characterize that can describe the similar characteristics of the signals. Therefore, multifractal dimension is used in this paper to extract the subtle features of different impulse noise signals, in order to achieve the purpose of the classification and identification of the radiation source.

\section{Introduction}

In recent years, individual radiation source identification $[1,2]$ has been developing rapidly as a nascent technology, which is an important topic in the field of noncooperative communication. Because of the continuous development of antireconnaissance technology and antijamming technology, we has found it difficult to get useful information through traditional intelligence information. The signal characteristics and individual subtle characteristics of individual radiation sources contain potential target information that is very meaningful. We can achieve the reconnaissance and surveillance of the goal by utilizing the subtle characteristics of the individual radiation sources. It is a new field with great research value. With the increasing complexity of the communication environment and the improvement of the information technology, the complexity of communication signals modulation types is becoming increasingly huge, while the differences between individuals radiation source are gradually decreasing. Therefore, the signal processing technology in recent years has become a hot issue in signal analysis areas and an integral part of signal recognition field. As the recognition results of modulation signals affect directly the restoration of the original signals, the automatic identification of communication signal plays a crucial role in the field of communication investigation [3], signal authentication [4], spectrum management [5], radio monitoring
[6], electronic warfare [7], interference identification [8], and so forth. It is an important technology in radio spectrum management and intelligent signal processing. The basic task of communication signal recognition is to identify the modulation of the signals and estimate the modulation parameters and other basic information under the noisy environment or the multiple signal transmission conditions, finally, providing adequate basis for further processing and analyzing the signals.

A signal estimation and recognition method under white noise environment is put forward in literature [9], which obtains a good recognition performance. Therefore, in this paper, multifractal dimension is used to extract the different distribution of additional subtle impulse noise characteristics of radiation source, so as to achieve the purpose of classification and recognition.

\section{Flowchart of Identification System}

For the communication individual radiation source, even two models from the same manufacturer, the same individual categories, and the same working conditions with the same operating parameters, there are also subtle differences between individual radiation signals, due to the individual differences in their internal components. Therefore, it is possible to use the subtle characteristics to identify the signals of communication individual radiation source. Nature 


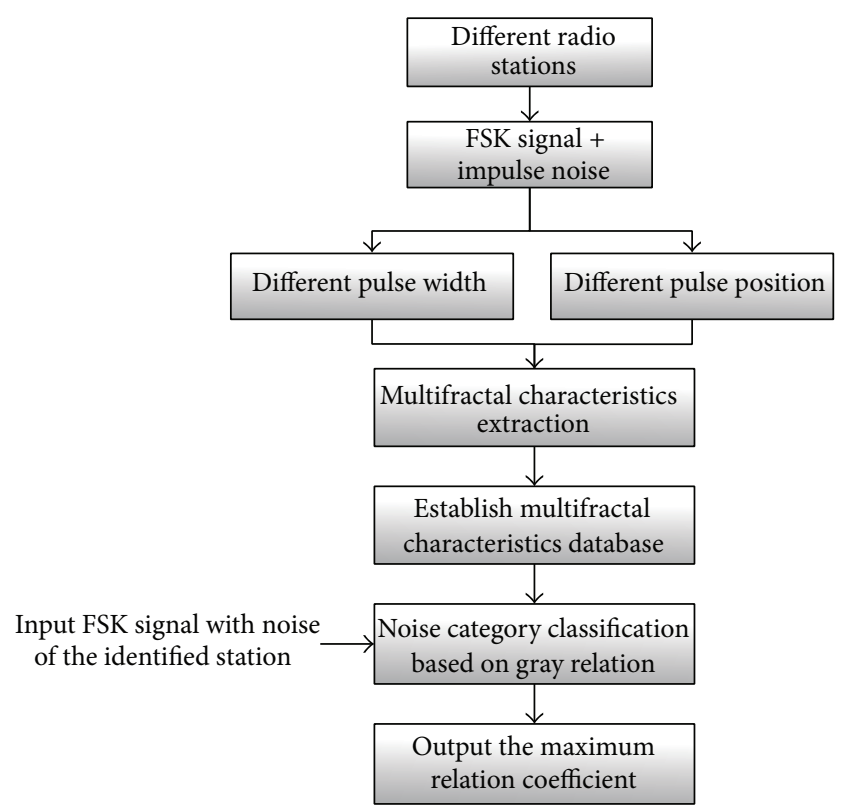

FIGURE 1: Flowchart of identification system based on multifractal dimension.

communications emitter individual identification is based on individual characteristics of radiation subtle signals, which is used to determine where radio signals come from. Furthermore, it can also identify the specific communication unit from the mixed signals accurately.

It is difficult to extract the subtle features from individual radiation source under low SNR. To solve this problem, we proposed the subtle characteristics extraction algorithm based on multifractal dimension. The specific identifying flowchart is shown in Figure 1.

First, the noises with different pulse widths and different pulse positions are added to different radiation sources which transmit the same signals. Then, the multifractal theory is used to extract the subtle characteristics of different signals and to establish the database of multiple fractal dimension features. Finally, multifractal dimension characteristics of the unknown signals which would be identified are obtained, and then gray relation value is calculated with the signal features in the database. The type of the radio in the database which has the maximum output relation degree is the type of the radiation source to be identified; thus the radiation source classification is realized.

\section{Basic Theory Analysis}

3.1. Multifractal Dimension Theory. Fractal dimension is an important indicator which is used to measure signals irregularity and explore the complexity of things. At present, it has been widely applied in image analysis [10], vibration signal fault diagnosis [11], and dynamics analysis [12]. Onedimensional fractal dimension, box-counting dimension [13], can be sufficient for the description of the features of a simple signal. But, for complex signals, multifractal parameters are needed to describe the local characteristics of different levels. Therefore, the concept of multifractal dimension [14] is introduced in order to further understand the local characteristic of the signal and extract the signal features of different levels complexity. The proposed multifractal theory makes the subtle feature extraction of individual radio source possible.

Multifractal dimension is used to describe signal characteristics of different levels, and it is also used to discuss the probability distribution of parameters. It divides the research object (set its linear degree to 1 ) into $N$ small areas. Suppose the line degree of areas $i$ is $\varepsilon_{i}$, and the density distribution function $P_{i}$ of area $i$ can be described by different scaling index $\alpha_{i}$ as

$$
P_{i}=\varepsilon_{i}^{\alpha_{i}}, \quad i=1,2, \ldots, N_{i} .
$$

Noninteger $\alpha_{i}$ can commonly be called a singular index, and its value is relevant to its region.

In order to get the distribution characteristics of a series of subsets, function $X_{q}(\varepsilon)$ is defined as follows:

$$
X_{q}(\varepsilon)=\sum_{i=1}^{N} P_{i}^{q} .
$$

It is the weighting summation of probability in each area. Further, the generalized fractal dimension $D_{q}$ is defined as

$$
D_{q}=\frac{1}{q-1} \lim _{\varepsilon \rightarrow 0} \frac{\ln X_{q}(\varepsilon)}{\ln \varepsilon}=\frac{1}{q-1} \lim _{\varepsilon \rightarrow 0} \frac{\ln \left(\sum_{i=1}^{N} P_{i}^{q}\right)}{\ln \varepsilon},
$$

where $X_{q}(\varepsilon)$ reflects the different effects of $P_{i}$. The formula shows that, when $q \gg 1$, large probability area plays the leading role in the summation formula $\sum_{i=1}^{N} P_{i}^{q}$ and, at the same time, $X_{q}(\varepsilon)$ and $D_{q}$ reflect the properties of big probability areas. Conversely, when $q \ll 1, X_{q}(\varepsilon)$ and $D_{q}$ reflect the properties of small probability areas (sparse areas). In this way, different $q$ values reflect the properties of different probability characteristic areas. Through the weighting summation processing, it divides the signal into many areas with different singular degrees, and, therefore, the internal structure of the signal is hierarchical. Calculate $P_{i}$ through phase space reconstruction and extract the different probability characteristics of the signal; the multilevel feature extraction results can be finally got in this paper.

3.2. Gray Relation Theory Analysis. Assume the system behavior sequence is

$$
\begin{aligned}
X_{0} & =\left(x_{0}(1), x_{0}(2), \ldots, x_{0}(n)\right), \\
X_{1} & =\left(x_{1}(1), x_{1}(2), \ldots, x_{1}(n)\right), \\
& \vdots \\
X_{i} & =\left(x_{i}(1), x_{i}(2), \ldots, x_{i}(n)\right), \\
X_{m} & =\left(x_{m}(1), x_{m}(2), \ldots, x_{m}(n)\right) .
\end{aligned}
$$


To the $\xi \in(0,1)$, we can get

$$
\begin{aligned}
& \gamma\left(x_{0}(k), x_{i}(k)\right) \\
& =\frac{\left(\min _{i} \min _{k}\left|x_{0}(k)-x_{i}(k)\right|+\xi \max _{i} \max _{k}\left|x_{0}(k)-x_{i}(k)\right|\right)}{\left(\left|x_{0}(k)-x_{i}(k)\right|+\xi \max _{i} \max _{k}\left|x_{0}(k)-x_{i}(k)\right|\right)},
\end{aligned}
$$

where $\varepsilon_{i}$ is defined as the distinguishing coefficient.

Then the grey relation is defined as

$$
\gamma\left(X_{0}, X_{i}\right)=\frac{1}{n} \sum_{k=1}^{n} \gamma\left(x_{0}(k), x_{i}(k)\right) .
$$

Here, $\gamma\left(X_{0}, X_{i}\right)$ is the grey relation of $X_{0}$ and $X_{i}$. The gray relational $\gamma\left(X_{0}, X_{i}\right)$ is often written as $\gamma_{0 i}$ and the correlative coefficient $\gamma\left(x_{0}(k), x_{i}(k)\right)$ of $k$ is written as $\gamma_{0 i}(k)$.

Thus the calculation procedure of grey correlation can be got as follows.

(1) Evaluate the initial value (or mean value) for each sequence, and set it as follows:

$$
X_{i}^{\prime}=\frac{X_{i}}{x_{i}(1)}=\left(x_{i}^{\prime}(1), x_{i}^{\prime}(2), \ldots, x_{i}^{\prime}(n)\right),
$$

where $i=0,1,2, \ldots, m$.

(2) Evaluate the $D$-value:

$$
\begin{gathered}
\Delta_{i}(k)=x_{0}^{\prime}(k)-x_{i}^{\prime}(k), \\
\Delta_{i}=\left(\Delta_{i}(1), \Delta_{i}(2), \ldots, \Delta_{i}(n)\right),
\end{gathered}
$$

where $i=0,1,2, \ldots, m$.

(3) Get the maximum and minimum values of $D$-value:

$$
\begin{aligned}
& M=\max _{i} \max _{k} \Delta_{i}(k), \\
& m=\min _{i} \min _{k} \Delta_{i}(k) .
\end{aligned}
$$

(4) Get the grey relational coefficient:

$$
\gamma_{0 i}(k)=\frac{m+\xi M}{\Delta_{i}(k)+\xi M}, \quad \xi \in(0,1),
$$

where $k=1,2, \ldots, n$ and $i=1,2, \ldots, m$.

(5) Calculate the gray correlation:

$$
\gamma_{0 i}=\frac{1}{n} \sum_{k=1}^{n} \gamma_{0 i}(k), \quad i=1,2, \ldots, m
$$

Thus the gray relation values of different features are obtained, and the category of the signals to be recognized can be judged by comparing the value of the gray relation degree.

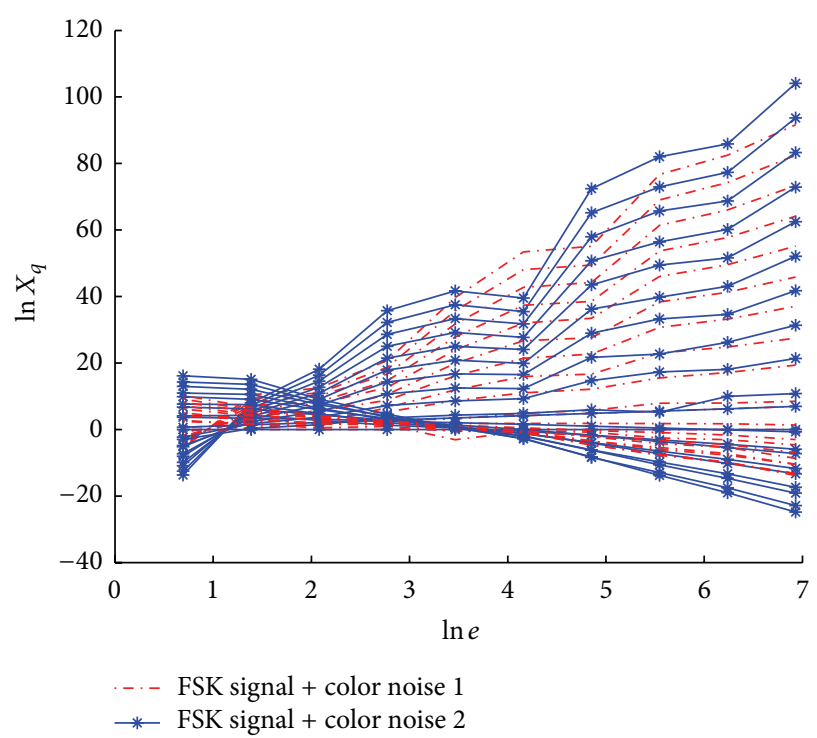

FIGURE 2: Multifractal curves of FSK signals mixed with different color noises.

\section{Simulation Results and Analysis}

In order to verify the property of multifractal dimension, we randomly generate 1000 FSK signal samples with MATLAB. In Monte Carlo simulation experiment, two kinds of color noises are added onto FSK signals. Taking the two color noises as example, we suppose it to be the output of the internal device subtle characteristics, figure out the multifractal dimension curves, and verify the performance of the proposed algorithm. The $q$ is varied from -10 to 10 , which is used to get 21 multifractal dimensions and reflect the 21 levels of different subtle features of the signal. As we can see from Figure 2, the abscissa represents the dimension of reconstructed phase space, denoted by $\ln e$, and the ordinate represents the natural logarithm of $\sum_{t=1}^{6} P_{t}^{q_{s}}$, denoted by $\ln X_{q}$. Simulation results are shown in Figure 2.

The simulation results show the following.

(1) The smaller the reconstructed phase space dimension is, the smaller the difference of different probability levels is. And it does not give further differences of the signals. When reconstructed phase space is larger, the signal is divided into different areas to study, and the probability of different characteristics areas is calculated, so more details of the signals are detected, and the differences are bigger.

(2) According to the fact that different $q$ values reflect the characteristics of different probability characteristic areas, the $q$ values of the curves take $-10-10$ from bottom to top, respectively. Thus it can be seen from the distribution of the curves that, when $q$ is bigger, the differences between the signals are much more obvious.

As the distributions of different color noises are different, multifractal dimension can be used to detect the complexity 


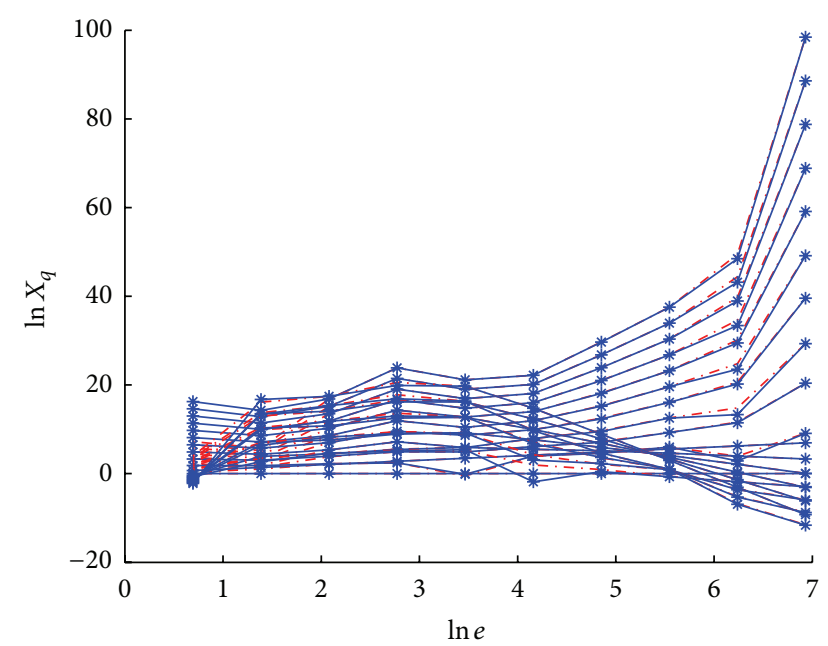

- - FSK signal + pulse noise 1

* FSK signal + pulse noise 2

FIGURE 3: Multifractal curves when the pulse positions of the noises are different.

changes of FSK signals, whose multifractal curves also show obvious differences. Then gray relation theory is used to identify the two kinds of color noise, and the results show the recognition rate of one hundred percent.

Taking FSK signal of communication individual radio source with the same distribution of impulse noise as an example, multifractal characteristics of FSK signal sequences with the same pulse distribution noise are discussed, but the subtle differences are that the pulse position and pulse width are not the same. Then the recognition performance of this algorithm is verified. The signal sequences consisted of 1024 points, where one signal produces an impulse noise at the place of 401-403 points, and the other signal sequences produce an impulse noise at the place of 701-703 points. Multifractal dimension curves are calculated and the simulation results are shown in Figure 3. The same signal sequences consist of 1024 points as described above, but, at the same place, the width of one impulse noise is 1 and the width of another impulse noise is 3 . And the multifractal dimension curves are calculated and the simulation results are shown in Figure 4.

Conclusions can be drawn from simulation results in Figures 3 and 4 as follows.

(1) When the pulse positions of the noises are different, the increase of reconstructed phase space dimension will fade the characteristic of the noise. Only under lower phase space dimensions are the multifractal dimensions of signals different to some extent.

(2) When $q$ value is small, the differences of multifractal dimensions are also small, which makes it hard to classify the signals. Therefore, the differences of large probability area signals are more obvious.

(3) The distribution curves show that the influence of the impulse noises with different widths is bigger than that of the impulse noises with different positions.

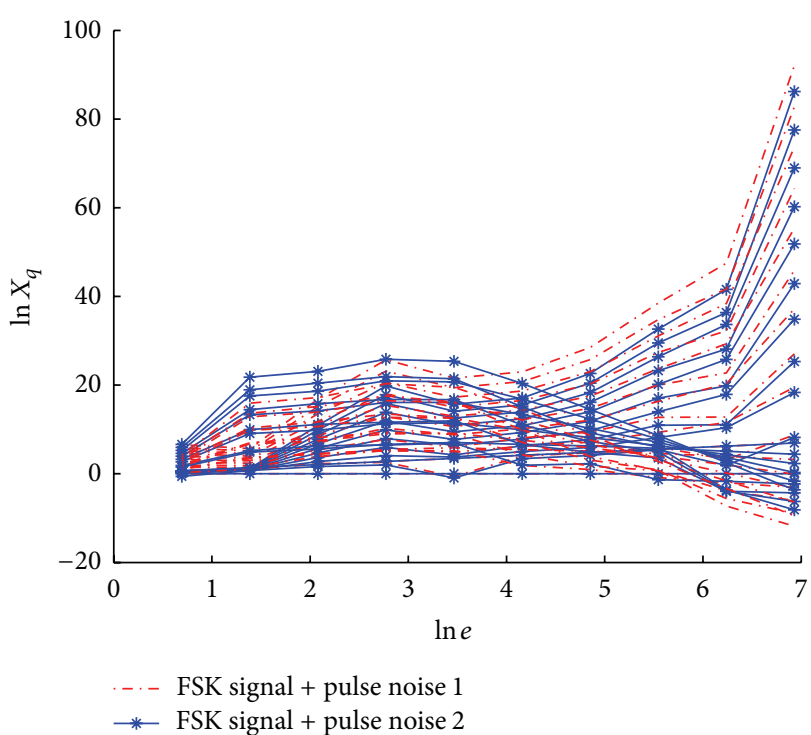

FIGURE 4: Multifractal curves when the pulse widths of the noises are different.

TABLE 1: Recognition rates of two kinds of feature extraction methods under the condition of different position pulse.

\begin{tabular}{lcc}
\hline Times & $\begin{array}{c}\text { Based on } \\
\text { multifractal } \\
\text { dimension }\end{array}$ & Based on entropy \\
\hline 1 & $100 \%$ & $95.5 \%$ \\
2 & $97.5 \%$ & $92.0 \%$ \\
3 & $96.0 \%$ & $80.0 \%$ \\
4 & $100 \%$ & $82.5 \%$ \\
5 & $99.0 \%$ & $79 \%$ \\
6 & $99.5 \%$ & $85.5 \%$ \\
\hline Average value & $98.7 \%$ & $85.8 \%$ \\
Variance & $3.58 \%$ & $14.9 \%$ \\
\hline
\end{tabular}

Since the additive noises are randomly generated, the noises will be different at each time; however, the pulse position and pulse width of the noises are the same. Therefore, it has better universality and application under the condition of the same pulse position and pulse width. As described before, 1000 samples are randomly generated, and Monte Carlo simulation experiment is carried out to calculate the recognition effect. Due to the randomness of the noise, there are some differences on noise distribution. Therefore, there are some differences of the recognition rates at each time. In this experiment, we carry out the simulation for 6 times and randomly generate 2000 distribution sequences of the two kinds of noises every time. Then we use gray relation theory to classify and identify the signals. The entropy features of the noises are extracted under the same background, and then the signals could be recognized. The recognition rates of the two kinds of methods are shown in Tables 1 and 2, and the comparison of the simulation time is shown in Table 3. 
TABLE 2: Recognition rates of two kinds of feature extraction methods under the condition of different pulse width.

\begin{tabular}{lcc}
\hline Times & $\begin{array}{c}\text { Based on } \\
\text { multifractal } \\
\text { dimension }\end{array}$ & Based on entropy \\
\hline 1 & $100 \%$ & $83.5 \%$ \\
2 & $98.5 \%$ & $92.0 \%$ \\
3 & $100 \%$ & $70.5 \%$ \\
4 & $98.0 \%$ & $73.0 \%$ \\
5 & $100 \%$ & $66.0 \%$ \\
6 & $98.5 \%$ & $92.5 \%$ \\
\hline Average value & $99.0 \%$ & $79.6 \%$ \\
Variance & $1.87 \%$ & $25.4 \%$ \\
\hline
\end{tabular}

TABLE 3: Recognition time of two feature extraction methods.

\begin{tabular}{lc}
\hline Recognition method & Simulation time $(\mathrm{ms})$ \\
\hline Based on multifractal dimension & 13.8 \\
Based on entropy & 10.1 \\
\hline
\end{tabular}

The comparison of Tables 1, 2, and 3 depicts that the results of using entropy feature method to measure the noises subtle characteristics are rougher than multifractal dimension. Therefore, the recognition rate is relatively lower compared with multifractal characteristics. Meanwhile, under the condition of randomly generated noise, the recognition rate of entropy feature is not stable, and the fluctuated variance is bigger. For the computational complexity, the algorithm of multifractal dimension is more complex than the algorithm of the entropy value. Furthermore, the computational complexity of multifractal dimensions is decided by the calculated dimension that we choose. We need to compute more dimensions if we want to achieve more accurate feature extraction. As a result, the amount of calculation will be greater. On the contrary, the amount of calculation will be less if we calculate fewer dimensions. Considering the better recognition rate and stability, multifractal characteristics have better application value in communication individual recognition.

\section{Conclusion}

It is difficult to extract the subtle features of the radio station under low SNR. In order to solve this problem, the paper proposes a subtle feature extraction algorithm based on multifractal dimension. First, for the same FSK signal of radio transmitter, different color noises are added to the signals to verify the feasibility of subtle features classification based on multifractal dimension. Then the FSK signals with different pulse widths and different pulse positions are used to imitate the different additional small internal radio noises, and multifractal dimension is used to classify the signals and verify the effect of recognition algorithm. Simulation results show that the multifractal dimension can characterize the small changes of signals in different scales to achieve the recognition of different radiation sources. It has great value in the project.

\section{Conflict of Interests}

The authors declare that there is no conflict of interests regarding the publication of this paper.

\section{Acknowledgments}

The authors are thankful to the support of the National Nature Science Foundation of China (no. 61301095 and no. 51374099), the Fundamental Research Funds for the Central Universities (no. HEUCF140807), the Heilongjiang Province Natural Science Foundation (no. F201345), and the Heilongjiang Province Natural Science Foundation for the Youth (no. QC2012C070).

\section{References}

[1] A. K. Nandi and E. E. Azzouz, "Algorithms for automatic modulation recognition of communication signals," IEEE Transactions on Communications, vol. 46, no. 4, pp. 431-436, 1998.

[2] Q. Shi and Y. Karasawa, "Automatic modulation identification based on the probability density function of signal phase," IEEE Transactions on Communications, vol. 60, no. 4, pp. 1033-1044, 2012.

[3] J. Chen, Z. Safar, and J. A. Sørensen, "Multimodal wireless networks: communication and surveillance on the same infrastructure," IEEE Transactions on Information Forensics and Security, vol. 2, no. 3, pp. 468-484, 2007.

[4] A. Krueger, "Valuation of water quality and consumer choice and recognition of beach eco-certification in Tampa Bay area beaches," in Proceedings of the MTS/IEEE OCEANS, pp. 941948, September 2005.

[5] J. Giesbrecht, "A monitoring tool for HF frequency management and license enforcement," in Proceedings of the 10th IET International Conference on Ionospheric Radio Systems and Techniques, pp. 263-267, 2006.

[6] H. Khoshnevis, "Pilot signature based detection of DVB-T2 broadcasting signal for cognitive radio," in Proceedings of the $3 r d$ International Conference on Network of the Future (NOF '12), pp. $1-5,2012$.

[7] Q. Liang, X. Cheng, and S. W. Samn, "NEW: network-enabled electronic warfare for target recognition," IEEE Transactions on Aerospace and Electronic Systems, vol. 46, no. 2, pp. 558-568, 2010.

[8] Y. Makimoto, H. Hiura, N. Goto, and S.-I. Yanagiya, "Waveguide-type optical circuit for recognition of optical QPSK coded labels in photonic router," Journal of Lightwave Technology, vol. 27, no. 1, pp. 60-67, 2009.

[9] B. Hu and R. G. Gosine, "New eigenstructure method for sinusoidal signal retrieval in white noise: estimation and pattern recognition," IEEE Transactions on Signal Processing, vol. 45, no. 12, pp. 3073-3083, 1997.

[10] M. Ivanovici and N. Richard, "Fractal dimension of color fractal images," IEEE Transactions on Image Processing, vol. 20, no. 1, pp. 227-235, 2011.

[11] B. Leprettre and J.-P. Navarre, "Three-component signal recognition using time, time-frequency, and polarization 
information-application to seismic detection of avalanches," IEEE Transactions on Signal Processing, vol. 46, no. 1, pp. 83-102, 1998.

[12] M. K. Roy, J. Paul, and S. Dattagupta, "Domain dynamics and fractal growth analysis in thin ferroelectric films," Journal of Applied Physics, vol. 108, no. 1, Article ID 014108, 2010.

[13] M. D. Prieto, A. G. Espinosa, J. R. R. Ruiz, J. C. Urresty, and J. A. Ortega, "Feature Extraction of demagnetization faults in permanent-magnet synchronous motors based on boxcounting fractal dimension," IEEE Transactions on Industrial Electronics, vol. 58, no. 5, pp. 1594-1605, 2011.

[14] G. Aklilu and J. M. Pitts, "Multi-scaling chaotic map suitable for modelling aggregate TCP/IP and MPEG4 traffic streams," Electronics Letters, vol. 41, no. 12, pp. 695-696, 2005. 


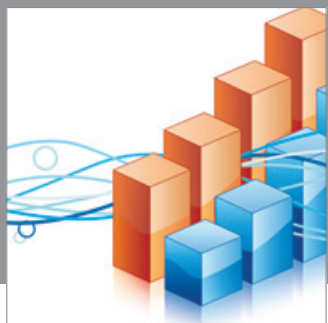

Advances in

Operations Research

mansans

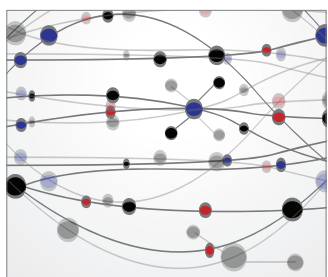

The Scientific World Journal
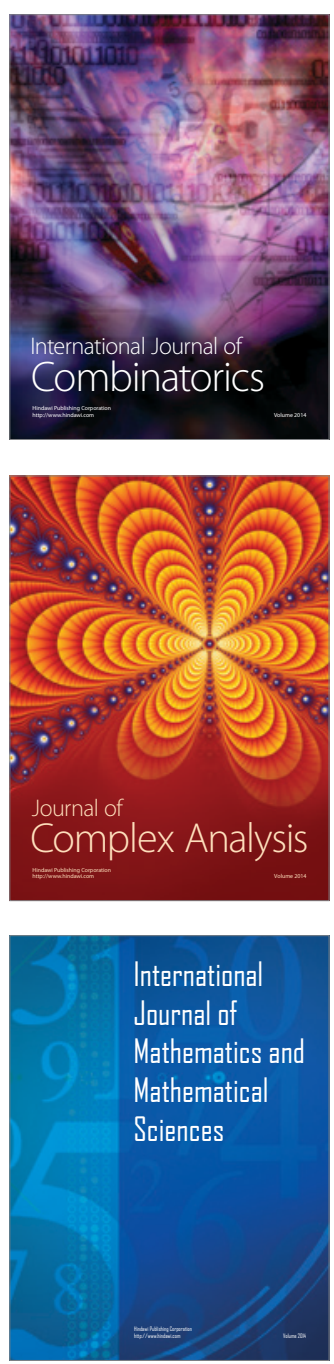
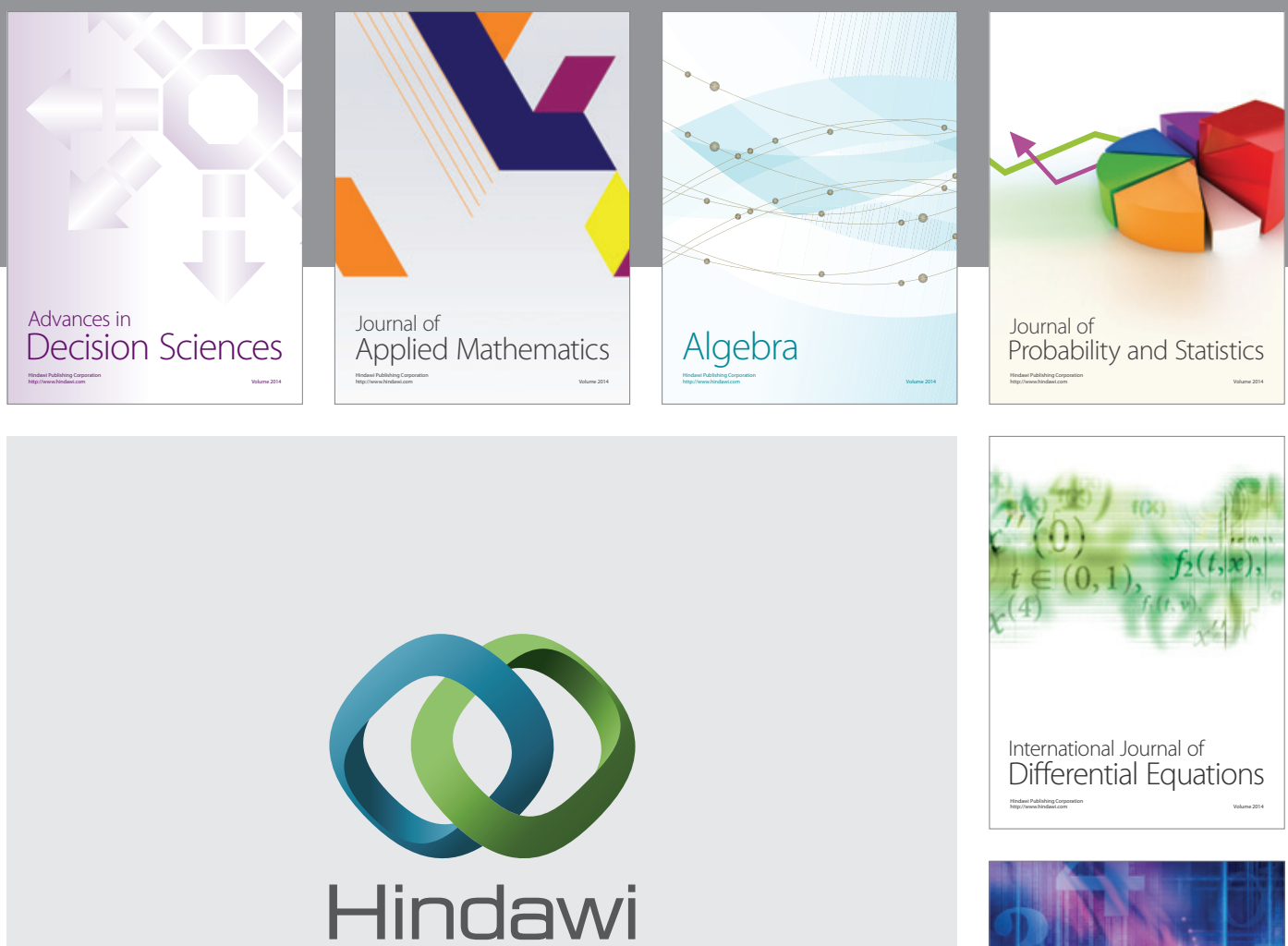

Submit your manuscripts at http://www.hindawi.com
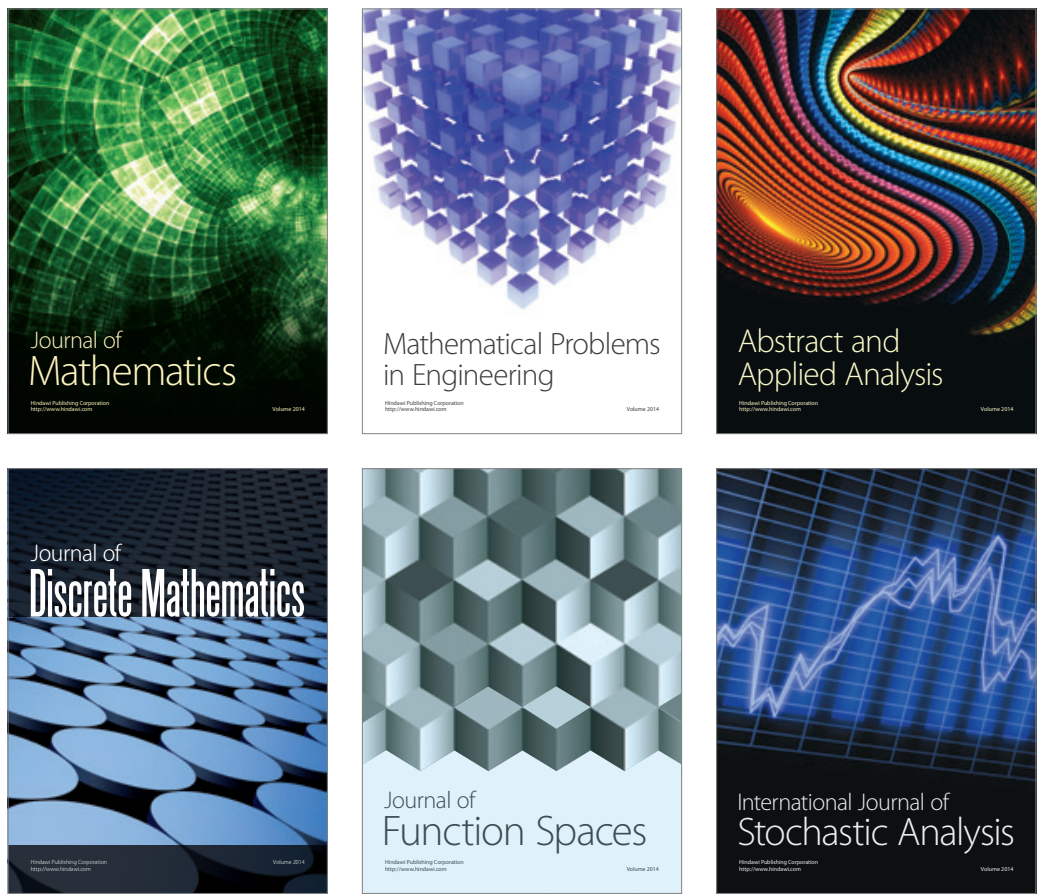

Journal of

Function Spaces

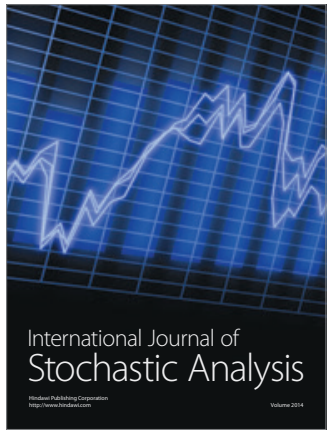

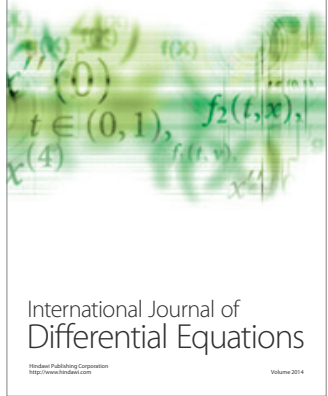
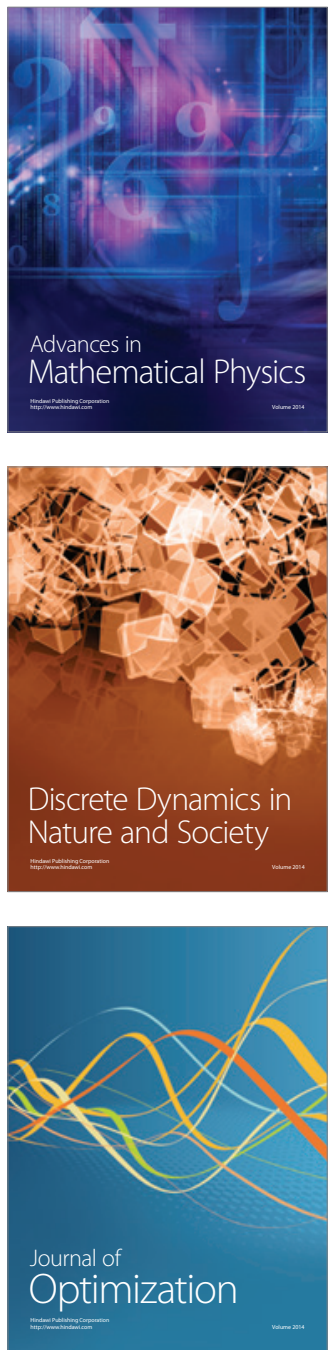